$$
\text { Ahmet BULUT }^{1 *} \text {, İbrahim BULUT }{ }^{2}
$$

ÖZET: Bu çalışmada, çok kısa yörünge dönemli W UMa türü örten çift sistemler KIC 7375612 ve KIC 9898401'nin 1şık eğrileri analizleri yapılarak, sistemlere ilişkin geometrik ve fiziksel parametreler ilk kez hesaplanmıştır. Işık eğrisi analizi için kullanılan gözlem verileri Kepler Örten Çiftler Kataloğundan alınmıştır. Işık eğrisi analizleri PHOEBE kodu kullanılarak yapılmıştır. Analizlerin sonuçlarına göre, KIC 7375612 ve KIC 9898401 'nin fotometrik kütle oranları sirasıyla $0.8059 \pm 0.0002,0.8103 \pm 0.0002$ ve yörünge eğimleri $60^{\circ} .18 \pm 0.05,67^{\circ} .17 \pm 0.11$ olarak elde edilmiştir. Sistemleri oluşturan bileşenlerin sicaklıkları KIC 7375612 için $6682 \mathrm{~K}$ ve $6672 \mathrm{~K}$, KIC 9898401 için ise $7376 \mathrm{~K}$ ve $7365 \mathrm{~K}$ olarak belirlenmiştir.

Anahtar Kelimeler: W UMa türü çift yıldız, Işık eğrisi analizi, Değen çift sistemler, KIC 7375612, KIC 9898401

\title{
Two Light Curves Analysis of W UMa-Type Eclipsing Binary Systems: KIC 7375612 and KIC 9898401
}

ABSTRACT: In this study, the light curves of the ultra-short period W UMa type KIC 7375612 and KIC 9898401 systems were analyzed for the geometric and physical parameters of the systems for the first time. Observation data for light curve analysis were taken from Kepler Eclipsing Binary Catalog. The light curves of KIC 7375612 and KIC 9898401 were analyzed by using PHOEBE code. According to the results, we calculated KIC 7375612 and KIC 9898401 of the photometric mass ratio $0.8059 \pm 0.0002$ and $0.8103 \pm 0.0002$, and inclination $60^{\circ} .18 \pm 0.05$ and $67^{\circ} .17 \pm 0.11$, respectively. The effective temperatures were obtained to be 6682 $\mathrm{K}$ and $6672 \mathrm{~K}$ for KIC 7375612 , to be $7376 \mathrm{~K}$ and $7365 \mathrm{~K}$ for KIC 9898401.

Keywords: W UMa type binary stars, Light curve analysis, Contact binary systems, KIC 7375612, KIC 9898401

\footnotetext{
${ }^{1}$ Ahmet BULUT (Orcid ID: 0000-0002-7215-926X), Çanakkale Onsekiz Mart Üniversitesi, Fen Edebiyat Fakültesi, Fizik Bölümü, Çanakkale, Türkiye

2ỉbrahim BULUT (Orcid ID: 0000-0002-7128-8887), Çanakkale Onsekiz Mart Üniversitesi, Fen Edebiyat Fakültesi, Uzay Bilimleri ve Teknolojileri Bölümü, Çanakkale, Türkiye

*Sorumlu Yazar/Corresponding Author: Ahmet BULUT, e-mail: abulut@comu.edu.tr
} 


\section{GÍRİŞ}

Çift yıldızlar üzerine yapılan çalışmalar, yıldız astrofiziğinde önemli bir yere sahiptir. $\mathrm{Bu}$ çalışmalar, kütle, yarıçap, ışınım gücü, sıcaklık gibi temel yıldız parametrelerinin doğrudan hesaplamasını sağladığı gibi yıldızlarda meydana gelen çok sayıda fiziksel olayın (manyetik etkiler, kütle aktarımı veya kaybı, açısal momentum aktarımı veya kaybı gibi) gözlenerek ve kuramsal modellerle test etmesini de sağlar. Son yıllarda sayısı gittikçe artan uydu teleskoplarıyla yapılan gözlemlerin analizleri sonucunda, yıldız parametrelerinin çok daha duyarlı belirlenmesine imkan verdi. Uydu teleskoplarından en önemlilerinden birisi olan Kepler Uydusu (Borucki ve ark., 2010: Batalha ve ark., 2010) görüş alanında yer alan yaklaşık 156000 tane yıldızın sürekli olarak gözlemini yapmıştır. Kepler uydusu, güneş sistemi dışı öte gezegenlerin keşfi için gerçekleştirilen uzun dönemli bir gözlem projesidir. Bu yıldızların içinden 2165 tanesi örten çift yıldız olarak belirlenmiş ve kataloglanmıştır (Slawson ve ark., 2011: Prsa ve ark 2011). Bu katalog, yıldızlarla ilgili bireysel ya da istatistiksel çalışmalar yapmak önemli bir kaynaktır.

W UMa türü örten çift yıldızlar ise, genelde her iki bileşen birbirine değme ve hemen hemen benzer iki yıldızdan oluşmuş sistemlerdir. Bu sistemlerde sistemi saran ortak bir atmosfer vardır. İki yıldızın fiziksel olarak birbirine değmesi nedeniyle birinden diğerine kütle ve 1şınım akışı mümkün olmaktadır. Bu çalışmada, Kepler kataloğunda yer alan çok kısa yörünge dönemli W UMa türü örten çift yıldız sistemleri olan KIC 7375612 ve KIC 9898401'nin 1şık eğrisi analizleri yapılarak fotometrik parametreleri belirlenmiştir. Rucinski (1992) ve Drake ve ark. (2014) tarafinda yörünge dönemi 0.22 gün altındaki sistemler çok kısa yörünge dönemli sistemler olarak adlandırılmıştır. KIC 7375612 (0.16 gün) ve KIC 9898401 (0.15 gün) sistemlerinin dönemleri bu limit değerin altındadır.

\section{MATERYAL VE YÖNTEM}

\section{Gözlem Verileri}

Bu çalışma için seçilen örten çift yıldızlar KIC 7375612 ve KIC 9898401 sistemleri hakkında temel bilgileri Çizelge 1'de verilmiştir. Bu sistemlerin analizlerinde kullanılacak fotometrik gözlem verileri Kepler veri tabanından kısa poz süreli (short cadance) gözlemlerden alınmıştır (Kepler Eclipsing Binaries, 2019). Her iki sistem için toplam 1000 gözlem noktası elde edilmiştir. Bu veriler ışı eğrisi analizinde kullanılmıştır.

Çizelge 1. Kepler kataloğundan alınan KIC 7375612 ve KIC 9898401 sistemleri için genel bilgiler

\begin{tabular}{cccccc}
\hline Sistemler & $\boldsymbol{\alpha}_{2000}(\mathbf{s a}$ dk sn) & $\boldsymbol{\delta}_{2000}\left({ }^{\circ}{ }^{\prime}\right.$ ") & V (kadir: m) & Tayf Türü & Yörünge Dönemleri (P) (gün) \\
\hline KIC 7375612 & 194152.071 & 425540.348 & 12.16 & A9III & 0.1600729 \\
KIC 9898401 & 193922.759 & 464215.047 & 12.22 & - & 0.1527742 \\
\hline
\end{tabular}

\section{Işık Eğrisi Analizi}

KIC 7375612 ve KIC 9898401 sistemlerinin 1şık eğrisi analizi, Wilson-Devinney (W-D) programina (Wilson ve Devinney, 1971) dayanan PHOEBE programı (Prsa ve Zwitter, 2005) kullanılarak yapılmıştır. W-D programı örten çift sistemine ilişkin geometrik ve fiziksel parametreleri diferansiyel düzeltme yoluyla hesaplar. Bu programa göre yıldızların yüzeyleri eş potansiyelli kabul edilir. Kuramsal ışık eğrisi; yörünge eğimi $(i)$, yörünge dış merkezliği $(e)$, enberi argümanı $(\omega)$, yüzey potansiyelleri $\left(\Omega_{1,2}\right)$, akı ağırlıklı ortalama yüzey sıcaklıkları $\left(T_{1,2}\right)$, kütle oranı $\left(q=m_{2} / m_{1}\right)$, normalize edilmemiş tek renk ışıtmaları $\left(L_{1,2}\right)$, lineer kenar kararma katsayıları $\left(x_{1,2}\right)$, çekim kararma üsleri $\left(g_{1,2}\right)$ ve bolometrik albedo $\left(A_{1,2}\right)$ parametrelerinin fonksiyonu olarak hesaplanır. 
Analizler için 1şık eğrileri, 0.25 ve 0.75 evrelerinde parlaklıkların ortalamalarına göre normalize edilmiştir. W-D programında yapılan analizler, değen çift sistemler için kullanılan Mod 3 seçilerek yapılmıştır. Bileşenlerin bazı parametreleri ışı eğrisi çözümü sırasında sabitlenmiştir. Her bir yıldızın doğrusal kenar kararma katsayıları Van Hamme (1993) tablolarından yıldızların sıcaklığına bağlı olarak belirlenmiştir. Çekim kararma üsleri $\left(g_{1,2}=0.32\right)$ ve bolometrik albedo katsayıları $\left(A_{1,2}=0.5\right)$ için, sistemlerin Kepler veri tabanında verilen sıcaklıklarına göre konvektif atmosfer kabulü altında Lucy (1968) tarafından önerilen değerler alınmıştır.

Kütle oranı $\left(q=m_{2} / m_{1}\right)$ W-D yönteminde önemli bir parametredir. Çünkü W-D yöntemi, kütle oranına duyarlı olan sistemin Roche geometrisine dayanmaktadır. KIC 7375612 ve KIC 9898401 sistemler için, kütle oranları için tayfsal olarak belirlenmiş bir değer bulunmadığından, ilk olarak sistemlere ilişkin $q$-taraması yapılmıştır. İşlem, $0.3 \leq q \leq 1.3$ aralığında olacak şekilde 0.05 arttırmayla seçilen $q$ değerleri sabit tutularak yapılmıştır. $\mathrm{Bu}$ işlem yapılırken yukarıda verilen girdi parametrelerinden $i, T_{2}, \Omega_{1}=\Omega_{2}$ ve $L_{1}$ parametreleri değişken seçilmiştir. $q$ taramasına ilişkin Şekil 1 ve Şekil 4'de verilmiştir. Böylece her iki sistemde de kütle oranı $\left(q=m_{2} / m_{l}\right)$ için yaklaşık 0.8 değerine ulaşılmıştır. $\mathrm{Bu}$ değer daha sonra programın diferansiyel düzeltmeler aşamasında kütle oranının başlangıç değeri olarak kullanılmıştır. $\mathrm{Bu}$ sistemlerin analizinde, Kepler veri tabanından birinci yıldızların etkin sıcaklıklarını $6682 \mathrm{~K}$ ve $7376 \mathrm{~K}$ olarak alınmıştır.

Analizlerde $i, q, T_{2}, \Omega_{1}=\Omega_{2}$ ve $L_{1}$ serbest bırakılan parametrelerdir. Işık eğrilerinin çözümlerinin sonuçları Çizelge 2'de verilmiştir. W-D programı ile hesaplanan diferansiyel düzeltmelerin standart sapmaları parametreler için hata olarak verilmiştir. Çizelge 2'de $r_{1,2}$ bileşenlerin ortalama kesirsel yarıçapları gösterilmektedir. KIC 7375612 ve KIC 9898401'ın kütle oranları sırasıyla $q=0.8059 \pm$ $0.0002, q=0.8103 \pm 0.0002$ olarak bulunmuştur. $V$ filtresindeki ş̧ık eğrilerinin modellemesinden elde edilen parametreler kullanılarak oluşturulan kuramsal 1şık eğrilerinin, gözlemsel 1şı eğrileriyle uyumu Şekil 2 ve Şekil 5'de verilmiştir. Hesaplanan parametrelerle çizilen sistemlerin Roche geometrileri ise Şekil 3 ve Şekil 6'da verilmiştir.

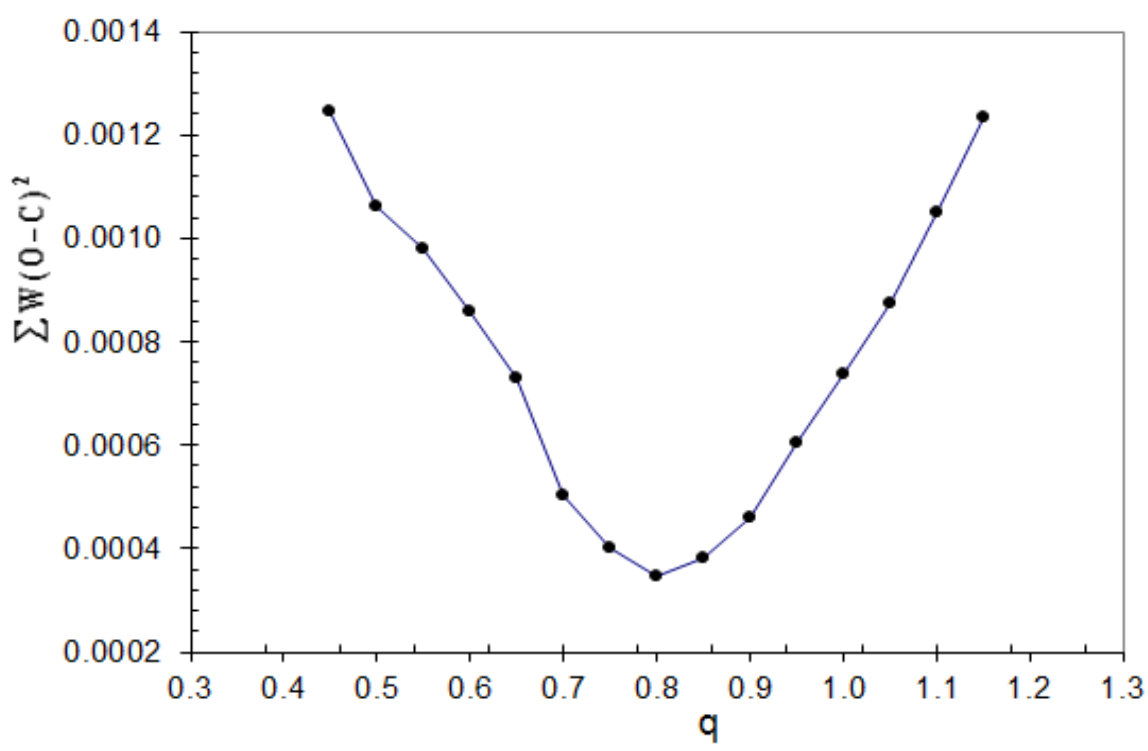

Şekil 1. KIC 7375612 sistemi için $q$-taraması 


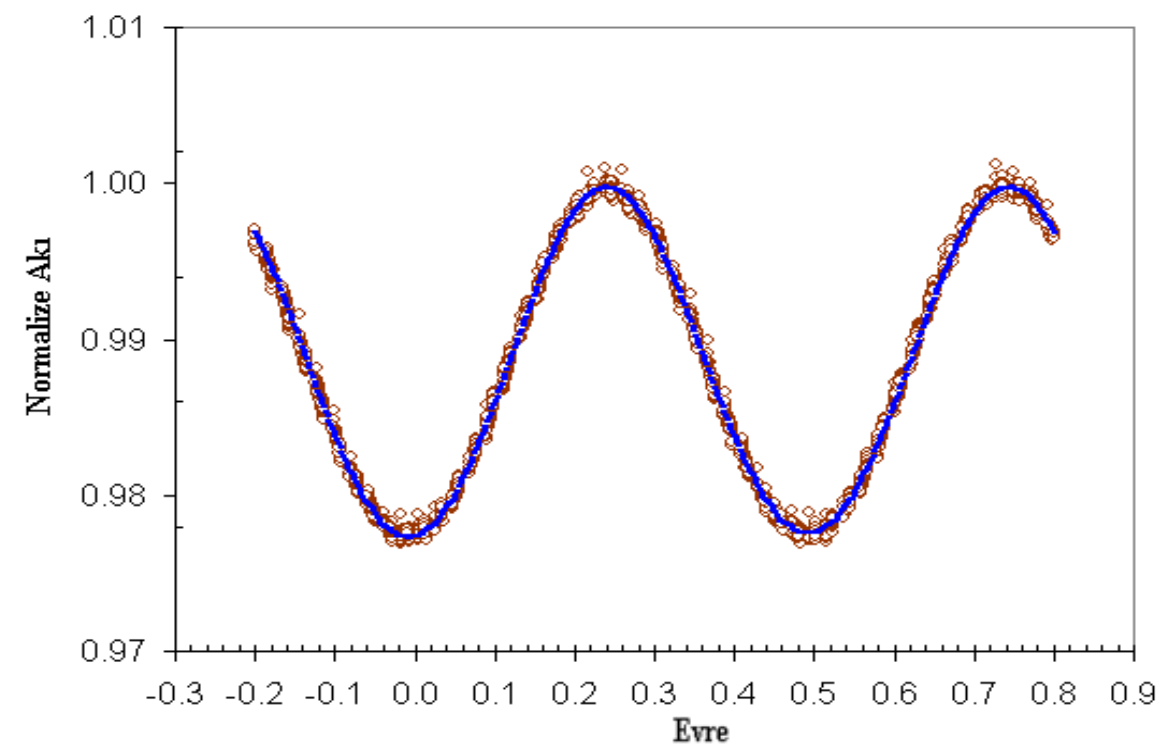

Şekil 2. KIC 7375612 sisteminin gözlemsel 1şık eğrisi ile kuramsal ış1k eğrisinin uyumu

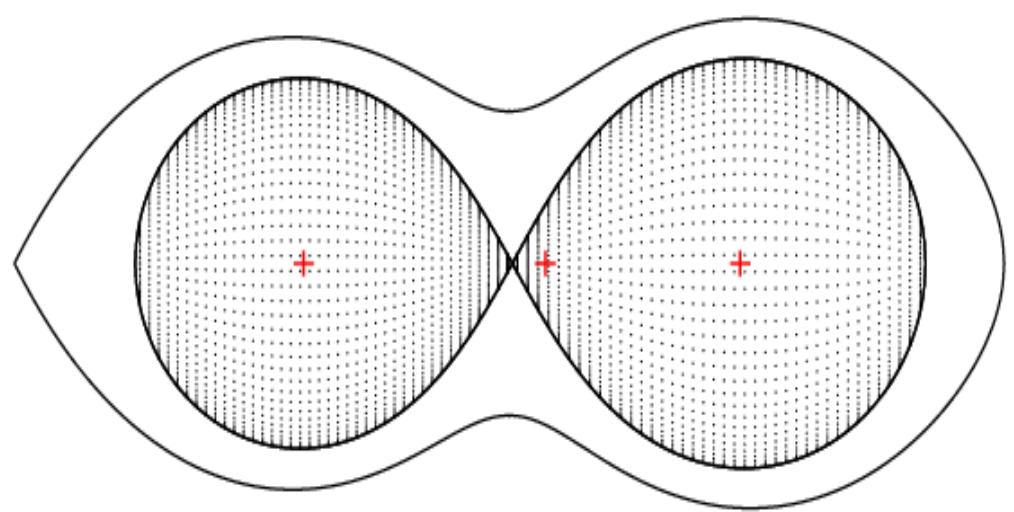

Şekil 3. KIC 7375612 sistemin Roche geometrisi $($ Evre $=0.25)$

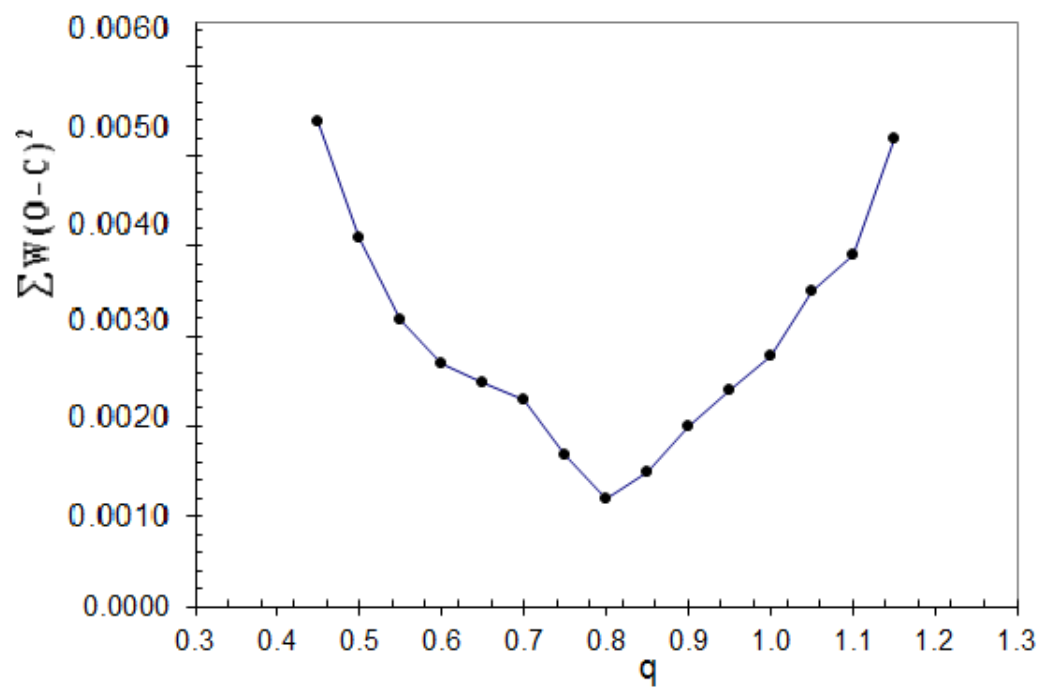

Şekil 4. KIC 9898401 sistemi için $q$-taraması 


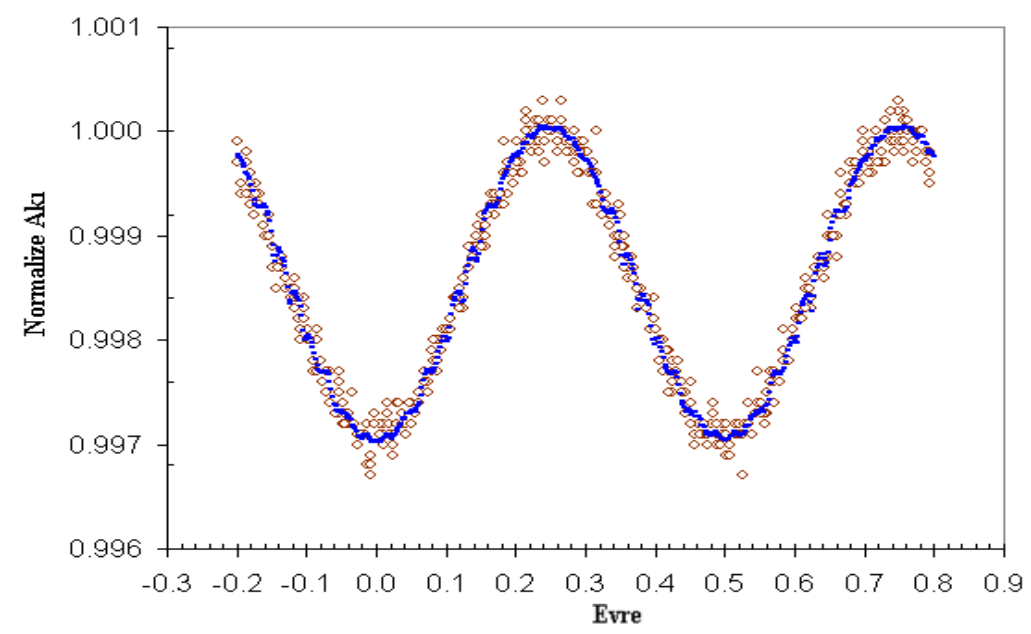

Şekil 5. KIC 9898401 sisteminin gözlemsel ışık eğrisi ile kuramsal 1şı eğrisinin uyumu

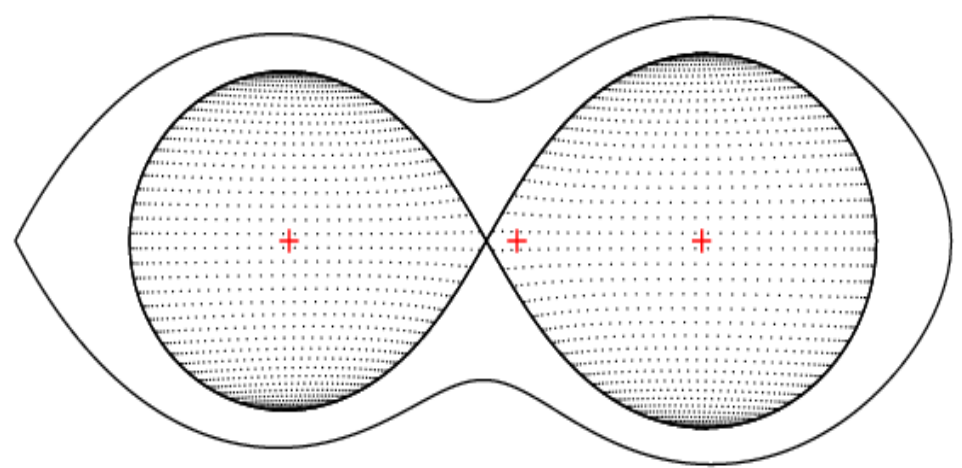

Şekil 6. KIC 9898401 sistemin Roche geometrisi $($ Evre $=0.25)$

Çizelge 2. KIC 7375612 ve KIC 9898401 sistemleri için yeni 1şı eğrisi analiz sonuçları

\begin{tabular}{|c|c|c|c|}
\hline Parametre & KIC 7375612 & KIC 9898401 & Açıklama \\
\hline$T_{0}(\mathrm{HJD})$ & 2454953.6387 & 2454964.7340 & Referans minimum zamanı \\
\hline$P($ gün$)$ & 0.1600729 & 0.1527742 & Yörünge dönemi \\
\hline$i$ (derece) & $60.18 \pm 0.05$ & $67.17 \pm 0.11$ & Eğim açıs1 \\
\hline$T_{l}(\mathrm{~K})$ & 6682 & 7376 & Yüzey sıcaklıkları \\
\hline$T_{2}(\mathrm{~K})$ & $6672 \pm 5$ & $7365 \pm 8$ & Yüzey sıcaklıkları \\
\hline$\Omega_{l=} \Omega_{2}$ & 4.6577 & 8.0607 & Yüzey potensiyelleri \\
\hline$q$ & $08059 \pm 0.0002$ & $0.8103 \pm 0.0002$ & Kütle oranı \\
\hline$x_{1}$ & 0.423 & 0.500 & Kenar kararma sabitleri \\
\hline$x_{2}$ & 0.422 & 0.500 & Kenar kararma sabitleri \\
\hline$A_{1}=A_{2}$ & 0.5 & 0.5 & Bolometrik albedolar \\
\hline$g_{1}=g_{2}$ & 0.32 & 0.32 & Çekim kararma üsleri \\
\hline$L_{1} /\left(L_{1}+L_{2}\right)$ & $05706 \pm 0.0012$ & $0.5859 \pm 0.0013$ & Kesirsel 1şınım güçleri \\
\hline$L_{2} /\left(L_{1}+L_{2}\right)$ & 0.42942 & 0.41401 & Kesirsel ışınım güçleri \\
\hline$r_{l}$ (kutup) & $0.258260 \pm 0.000012$ & $0.137853 \pm 0.000004$ & Birinci bileşenin kesirsel yarıçapı \\
\hline$r_{l}$ (kenar) & $0.262434 \pm 0.000017$ & $0.138181 \pm 0.000004$ & Birinci bileşenin kesirsel yarıçapı \\
\hline$r_{l}($ arka $)$ & $0.267701 \pm 0.000015$ & $0.138592 \pm 0.000004$ & Birinci bileşenin kesirsel yarıçapı \\
\hline$r_{2}$ (kutup) & $0.226143 \pm 0.000014$ & $0.116381 \pm 0.000004$ & İkinci bileşenin kesirsel yarıçapı \\
\hline$r_{2}($ kenar) & $0.229140 \pm 0.000015$ & $0.116586 \pm 0.000004$ & İkinci bileşenin kesirsel yarıçapı \\
\hline$r_{2}($ arka $)$ & $0.233867 \pm 0.000017$ & $0.116906 \pm 0.000004$ & İkinci bileşenin kesirsel yarıçapı \\
\hline$r_{l}($ ortalama $)$ & $0.262798 \pm 0.000015$ & $0.138209 \pm 0.000004$ & Ortalama kesirsel yarıçaplar \\
\hline$r_{2}($ ortalama $)$ & $0.229717 \pm 0.000015$ & $0.116624 \pm 0.000004$ & Ortalama kesirsel yarıçaplar \\
\hline
\end{tabular}




\section{BULGULAR VE TARTIŞMA}

\section{Mutlak Parametreler}

Radyal hız verilerinin olmaması nedeniyle, her iki sistemin mutlak parametrelerini belirlemek için (Dimitrov ve Kjurkchieva, 2015)'nin $P<0.27$ gün'e sahip sistemler yönelik verdiği yörünge dönemi - yarı büyük eksen uzunluğu $(P-a)$ ilişkisi kullanıldı.

$$
a=-1.154+14.633 x P-10.319 x P^{2}
$$

burada, $P$ gün biriminde, $a$ ise güneş yarıçapı birimindedir. Bu ifadeyle sistemlerin bir yarı büyük ekseni $(a)\left(R_{\odot}\right)$ hesaplandı. Burada $\odot$ sembolü Güneşi temsil etmektedir. Yine kısa dönemli çift sistemler için aşağı da "dönem-kütle" ilişkisi

$$
M=0.0134 / P^{2} x\left(-1.154+14.633 x P-10.319 x P^{2}\right)^{3}
$$

kullanılarak $M$, ikilinin toplam kütlesi hesaplandı (Dimitrov ve Kjurkchieva, 2015). Bileşenlerin ortalama kesirsel yarıçapları aşağıdaki formülle elde edildi.

$$
r_{1,2 \text { ortalama }}=\left(r_{\text {kutup }}+r_{\text {kenar }}+r_{\text {arka }}\right)^{1 / 3}
$$

(1)’den hesaplanan yarı büyük eksen değerleri kullanarak bileşenlerin yarıçapları

$$
R_{1,2}=\operatorname{ar}_{1,2 \text { ortalama }}
$$

eşitliği kullanılarak hesaplanmıştır. Bileşenlerin kütleleri ise

$$
\begin{aligned}
& q=M_{1} / M_{2} \\
& M_{1}+M_{2}=\frac{a^{3}}{p^{2}}
\end{aligned}
$$

bağıntıları kullanılarak hesaplanmıştır. Güneşin sıcaklığı $T_{\odot}=5780 \mathrm{~K}$ olarak alınarak, bileşenlerin bolometrik mutlak parlaklıklar ise aşağıda verilen eşitlikler kullanılarak hesaplanmıştır.

$$
\begin{aligned}
& \operatorname{Mbol}_{1,2}=4.77-5 \log \left(R_{1,2} / R_{\Theta}\right)-10 \log \left(T_{1,2} / T_{\Theta}\right) \\
& L_{1,2}=\left(R_{1,2} / R_{\Theta}\right)^{2} x\left(T_{1,2} / T_{\Theta}\right)
\end{aligned}
$$

Yukarıda belirtilen adımlarla hesaplanan mutlak parametrelere ilişkin sonuçlar Çizelge 3'de verilmiştir.

\section{SONUÇ}

Bu çalışmada, Kepler Örten Çiftler Kataloğunda yer alan çok kısa yörünge dönemli W UMa türü örten çift yıldız sistemlerinden seçilen KIC 7375612 ve KIC 9898401 'nin Kepler 1şık eğrileri WilsonDevinney yöntemi kullanarak ilk kez analiz edilmiştir. Her iki örten çift sisteme ilişkin geometrik ve fiziksel parametreler elde edilmiş ve sonuçlar Çizelge 2'de verilmiştir. Işık eğrilerinde belirgin bir 
O'Connell etkisi gözlenmemiştir. Elde edilen değerlere göre her iki sisteminde A alt tipi W UMa olduğu görülmüştür. KIC 7375612 ve KIC 9898401'in fotometrik kütle oranları sirasıyla $q=0.8059 \pm$ 0.0002 ve $q=0.8103 \pm 0.0002$ olarak bulunmuştur. Yüzey potansiyel değerleri kullanılarak sistemlere ait Roche modeli konfigürasyonları çizilmiştir. Sonuçlara göre her iki sistemde de bileşenler Roche Loblarının yaklaşık \%100 doldurduğu görülmüştür. Şu ana kadar bu sistemler için literatürde tayfsal çalışmalar bulunmamaktadır. Ancak, mutlak parametreler Dimitrov ve Kjurkchieva'nin (2015) kısa yörünge dönemli çift sistemler için verdiği ifadeler kullanılarak hesaplanmış ve Çizelge 3'de verilmiştir.

Her iki sistemde de mevcut gözlem aralığının yeterli olmaması nedeniyle $(O-C)$ eğrilerinde anlamlı bir değişim gözlenmemiştir. Ancak gelecekte yapılacak gözlemler, sistemlerdeki olası dönem değişimlerinin incelenmesine imkan sunabileceği düşünüldüğünde önemli olacaktır.

Çizelge 3. KIC 7375612 ve KIC 9898401 sistemleri için bu çalışmada hesaplanan mutlak parametreleri

\begin{tabular}{lccc}
\hline Parametre & Açıklama & KIC 7375612 & KIC 9898401 \\
\hline$a\left(R_{\odot}\right)$ & Yarı büyük eksen uzunluğu & 0.924 & 0.841 \\
$M_{\text {toplam }}\left(M_{\odot}\right)$ & Toplam kütle & 0.412 & 0.341 \\
$M_{1}\left(M_{\odot}\right)$ & Birinci bileşenin kütlesi & 0.228 & 0.188 \\
$M_{2}\left(M_{\odot}\right)$ & İkinci bileşenin kütlesi & 0.184 & 0.153 \\
$R_{1}\left(R_{\odot}\right)$ & Birinci bileşenin yarıçapı & 0.242 & 0.116 \\
$R_{2}\left(R_{\odot}\right)$ & İkinci bileşenin yarıçapı & 0.211 & 0.098 \\
$L_{1}\left(L_{\odot}\right)$ & Birinci bileşenin yüzey potansiyel & 0.105 & 0.036 \\
$L_{2}\left(L_{\odot}\right)$ & İkinci bileşenin yüzey potansiyel & 0.079 & 0.025 \\
$M_{\text {bol } 1}$ & Kadir & 7.22 & 8.38 \\
$M_{\text {bol } 2}$ & Kadir & 7.53 & 8.77 \\
\hline
\end{tabular}

\section{TEŞEKKÜR}

$\mathrm{Bu}$ makalede Kepler Uydusu tarafinda elde edilen veriler kullanılmıştır. Ayrıca, araştırmada, "NASA Astrophysics Data System Abstract Service" ve "SIMBAD" veri tabanının kaynakları kullanılmıştır.

\section{KAYNAKLAR}

Batalha NM, Borucki WJ, Koch DG, Bryson ST, Haas MR, Brown TM, Caldwell DA, Hall JR, Gilliland RL, Latham DW, Meibom S, Monet DG, 2010. Selection, Prioritization, and Characteristics of Kepler Target Stars. The Astrophysical Journal Letters, 713 (2): 109-114.

Borucki WJ, Koch D, Basri G, Batalha N, Brown T, Caldwell D, Caldwell J, Christensen-Dalsgaard J, Cochran WD, Devore E, Dunham EW, Dupree AK, Gautier TN, Geary JC, Gilliland R, Gould A, Howell SB, Jenkins JM, Kondo Y, Latham DW, Marcy GW, Meibom S, Kjeldsen H, Lissauer JJ, Monet DG, Morrison D, Sasselov D, Tarter J, Boss A, Brownlee D, Owen T, Buzasi D, Charbonneau D, Doyle L, Fortney J, Ford EB, Holman MJ, Seager S, Steffen JH, Welsh WF, Rowe J, Anderson H, Buchhave L, Ciardi D, Walkowicz L, Sherry W, Horch E, Isaacson H, Everett ME, Fischer D, Torres G, Johnson JA, Endl M, MacQueen P, Bryson ST, Dotson J, Haas M, Kolodziejczak J, Van Cleve J, Chandrasekaran H, Twicken JD, Quintana EV, Clarke BD, Allen C, Li J, Wu H, Tenenbaum P, Verner E, Bruhweiler F, Barnes J, Prsa A, 2010. Kepler Planet-Detection Mission: Introduction and First Results. Science, 327 (5968): 977-980. 
Dimitrov DP, Kjurkchieva DP, 2015. Ultrashort-period main-sequence eclipsing systems: new observations and light-curve solutions of six NSVS binaries. Monthly Notices of the Royal Astronomical Society, 448 (3): 2890-2899.

Drake AJ, Djorgovski SG, Garcia-Alvarez D, Graham MJ, Catelan M, Mahabal AA, Donalek C, Prieto JL, Torrealba G, Abraham S, Williams R, Larson S, Christensen E, 2014. Ultra-short Period Binaries from the Catalina Surveys. The Astrophysical Journal, 790 (2): 1-13.

Kepler Eclipsing Binaries, 2019. http://keplerebs.villanova.edu/ (Erişim Tarihi: 09.07.2020).

Lucy LB, 1968. The Structure of Contact Binaries. Astrophysical Journal, 151: 1123-1135.

Prsa A, Batalha N, Slawson RW, Doyle LR, Welsh WF, Orosz JA, Seager S, Rucker M, Mjaseth K, Engle SG, Conroy K, Jenkins J, Caldwell D, Koch D, Borucki W, 2011. Kepler Eclipsing Binary Stars. I. Catalog and Principal Characterization of 1879 Eclipsing Binaries in the First Data Release. The Astronomical Journal, 141 (3): 1-16.

Prsa A, Zwitter T, 2005. A Computational Guide to Physics of Eclipsing Binaries. I. Demonstrations and Perspectives. The Astrophysical Journal, 628 (1): 426-438.

Rucinski SM, 1992. Can Full Convection Explain the Observed Short-Period Limit of the W UMa-Type Binaries?. Astronomical Journal, 103: 960-966.

Slawson RW, Prsa A, Welsh WF, Orosz JA, Rucker M, Batalha M, Doyle LR, Engle SG, Conroy K, Coughlin J, Gregg TA, Fetherolf T, Short DR, Windmiller G, Fabrycky DC, Howell SB, Jenkins JM, Uddin K, Mullally F, Seader SE, Thompson SE, Sanderfer DT, Borucki W, Koch D, 2011. Kepler Eclipsing Binary Stars. II. 2165 Eclipsing Binaries in the Second Data Release. The Astronomical Journal, 142 (5): 1-14.

Van Hamme W, 1993. New limb-darkening coefficients for modeling binary star light curves. Astronomical Journal, 106 (5): 2096-2117.

Wilson RE, Devinney EJ, 1971. Realization of Accurate Close-Binary Light Curves: Application to MR Cygni. Astrophysical Journal, 166: 605-619. 